\title{
A quick-frozen section diagnosis of a lung tumor in a patient with a gastric cancer history
}

\author{
Takashi Yamashita $^{1}$, Kazuhito Funai ${ }^{1}$, Akikazu Kawase ${ }^{1}$, Hiroki Mori ${ }^{2}$, Satoshi Baba ${ }^{3}$, Norihiko \\ Shiiya $^{1}$, Haruhiko Sugimura ${ }^{3}$ \\ 1. First Department of Surgery, Hamamatsu University School of Medicine, Hamamatsu, Japan. 2. Department of Clinical \\ Pathology, Hamamatsu Medical Center, Hamamatsu, Japan. 3. Department of Diagnostic Pathology, Hamamatsu \\ University School of Medicine, Hamamatsu, Japan.
}

Correspondence: Kazuhito Funai. Address: 1-20-1, Handayama, Higashi, Hamamatsu, Shizuoka 431-3192, Japan. E-mail: kfunai@hama-med.ac.jp

Received: November 13, 2014

Accepted: February 5, 2015

Online Published: February 13, 2015

DOI : $10.5430 /$ crcp.v2n2p83

URL: http://dx.doi.org/10.5430/crcp.v2n2p83

\begin{abstract}
Even in the era of genome-based medicine, daily practices of surgeons and pathologists are full of challenges in making the best management in the circumstances where limited information is available. In this anecdotal experience we'd like to present an instructive case of the patient with past history of distal gastrectomy for gastric cancer, who was pointed out having a synchronous tumorous lesion in the lung during the surveillance. Scrutinized pathological report of the past gastric cancer, staging based on the Japanese Classification Systems, was T2 without lymphatic permeation, and the attending pathologist who saw the intraoperative frozen section showing a mucinous adenocarcinoma had been preoccupied with the metastatic gastric adenocarcinoma and answered so to the surgical team during the operation. Partial resection of the lung is suitable for metastatic tumor, therefore no further procedure was performed. Actually, it was not the metastatic case retrospectively and he needed additional appropriate procedure; lobectomy. We expound the complexities of this case in the hindsight and extract a lesson to both surgeons and pathologists.
\end{abstract}

\section{Key words}

Intra-operative diagnosis, Quick frozen tissue, Immunohistochemistry, Gastric cancer, Lung cancer, Metastasis

\section{I ntroduction}

In radiological surveillance of human lung in practice, we daily encounter various "suspicious" foci including primary and metastatic malignancies, benign tumors, and infectious lesions. In any modern countries, the first touch clinicians are required to ascertain the lesions accurately and proceed to the next acceptable step of treatments with correct definitive diagnosis toward the most suitable treatment. Though diagnostic imaging-modalities even in daily practice drastically improved, clinicians still make their therapeutic decisions based on microscopic "final” diagnosis.

So intraoperative pathological diagnosis plays a very important role in determinations among the options of treatment strategies. Actually in the field of thoracic surgery, it is sometimes essential to know the pathological characteristics of the lesion of the lung at the moment of the operation. For example, when the resected lesion is preoperatively suspected to be malignant and the intraoperative diagnosis of primary lung carcinoma was made, we should perform subsequent 
lobectomy and lymph node dissection as a standard procedure. On the other hand, when the lesion is a metastatic tumor, a more limited procedure, local resection is recommended, because lobectomy is a too invasive procedure for some patients, especially for cancer-carrying patients who are in poor general condition. These patients may be unable to undergo effective systemic chemotherapy against recurrence of carrying cancer due to operative invasion for lung, and the procedure may eventually shorten their survival time.

Intraoperative rapid diagnosis is performed in a limited time because the patient is under general anesthesia. This procedure is always a challenge for attending pathologists because the clinical requests are always demanding; that is, the shorter turn around time (usually a few minutes is ideal) and the more and more accurate qualitative diagnosis. Even though there are several progresses in equipment and methodology of intraoperative quick frozen diagnosis, there are many issues to overcome, thus the consensus among pathologists and surgeons is that rapid diagnosis based on frozen section is not a perfect method to diagnose even now. Although there is no reports on false-positive calling rates on the existence of malignancy by frozen section, false-negative occurs $1.1 \%-4.0 \%{ }^{[1,2]}$. In addition, in terms of tumor detected in the lung, it is sometimes very difficult or nearly impossible to determine the primary site of malignant tumor.

Here we report a case of a patient with lung tumor, in which the clinical decision and course were greatly influenced by the intraoperative rapid diagnosis and subsequent corrected permanent diagnosis. Our experience highlights the difficulty and challenges in rapid and qualitative diagnosis of lung tumors in a patient having past history of malignancies of other organs, and will provide a lesson to the surgeons in this field.

\section{Case presentation}

A 67-year-old male was consulted to our department of thoracic surgery because a solitary lung tumor was incidentally found during a preoperative work-up of gastric cancer in July 2013. His Brinkman index score was 1,870. First of all, laparoscopic distal gastrectomy and lymph node dissection was performed in August 2013. Pathological diagnosis and staging of the gastric cancer was a well-differentiated tubular adenocarcinoma of the stomach, pT2(MP), int, INFb, ly0, v0, N0, stage IB according to the Japanese Classification Systems ${ }^{[3]}$. Further CT examination delineated a solitary pulmonary nodule near visceral pleura of right S2, with $13 \mathrm{~mm} \times 12 \mathrm{~mm}$ in size, and associated spiculations. The diagnosis was primary lung adenocarcinoma based on CT appearance. In September 2013, the operation of lung was performed.

At operation, the nodule was wedge resected and submitted to the Diagnostic Pathology Division for intraoperative rapid diagnosis. Unexpectedly, a diagnosis of metastatic adenocarcinoma from the stomach was made. Under the doubt of this diagnostic response, because our experiences taught us it is extremely rare that metastatic lesion in the lung from gastric cancer appear as a solitary nodule like in this case ${ }^{[4]}$. We dissected \#11s lymph node and submitted it to the Diagnostic Pathology Division again for confirmation. Pathologists also found mucin-producing adenocarcinoma in the lymph node, and they re-confirmed their interpretation as a metastatic lesion from gastric cancer. As described above, a further procedure, lobectomy is not indicated for metastatic carcinoma, we have completed the surgery (see Figure 1).

A few days later, final pathological diagnosis came out after extensive immunostaining. It showed positive of TTF-1 and napsin A (see Figure 2, 3).

A conclusion was the primary colloid carcinoma of the lung, pT1bN1M0 stage IIA according to the latest guideline of WHO classification ${ }^{[5]}$. In the hindsight, if this immunohistopathological information had been available at the operation, a more appropriate procedure for this patient would be a lobectomy and lymph node dissection. Moreover the pathological finding based on the permanent section showed that the tumor spread near the cut-end microscopically. Thus we persuaded the patient to have an additional resection, but he declined our continuous recommendation, and he went back to the work. 


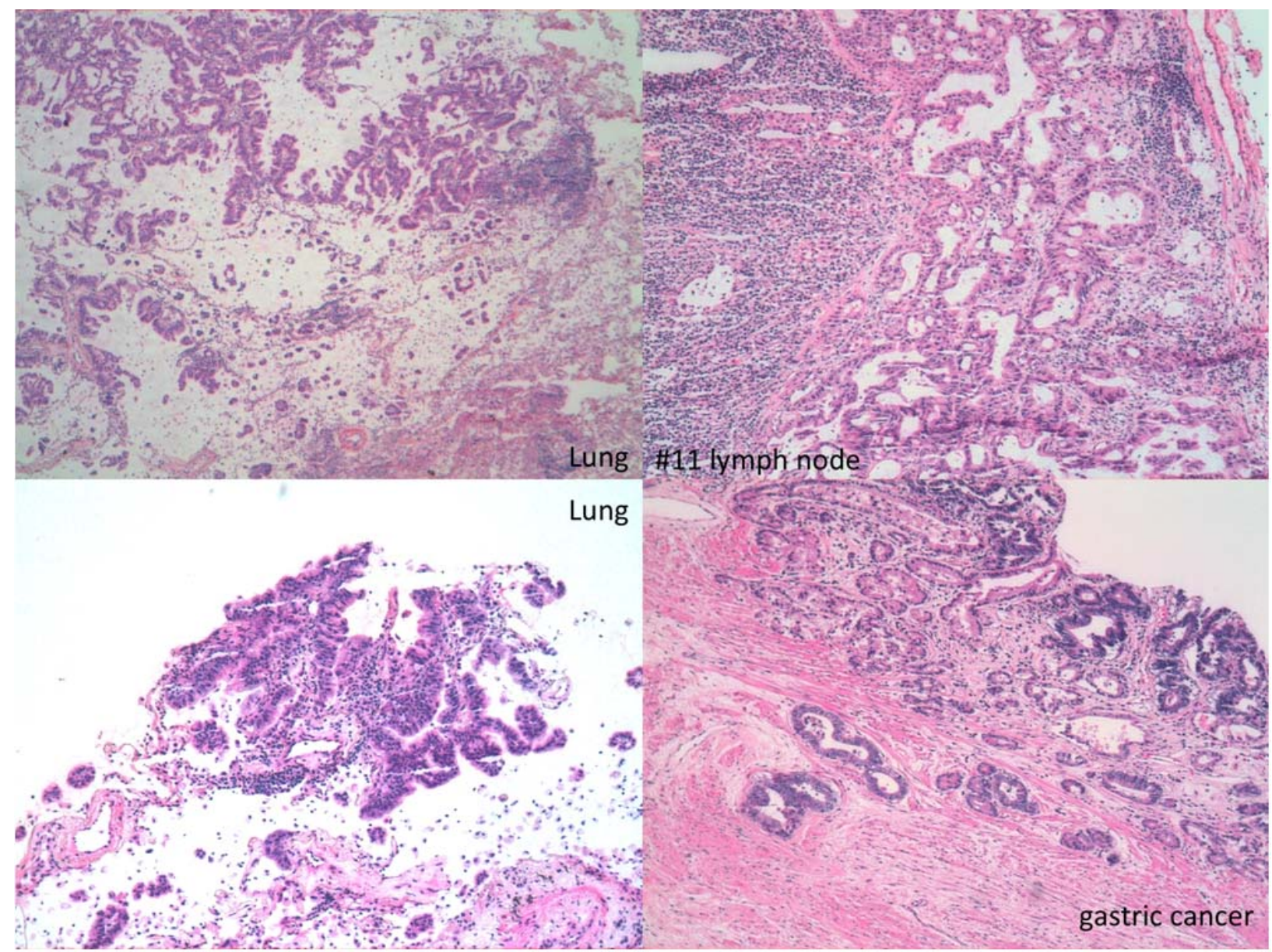

Figure 1. HE staining of the resected pulmonary nodule, \#11 lymph node, and gastric cancer

Figure 2. TTF-1 staining of the resected pulmonary nodule

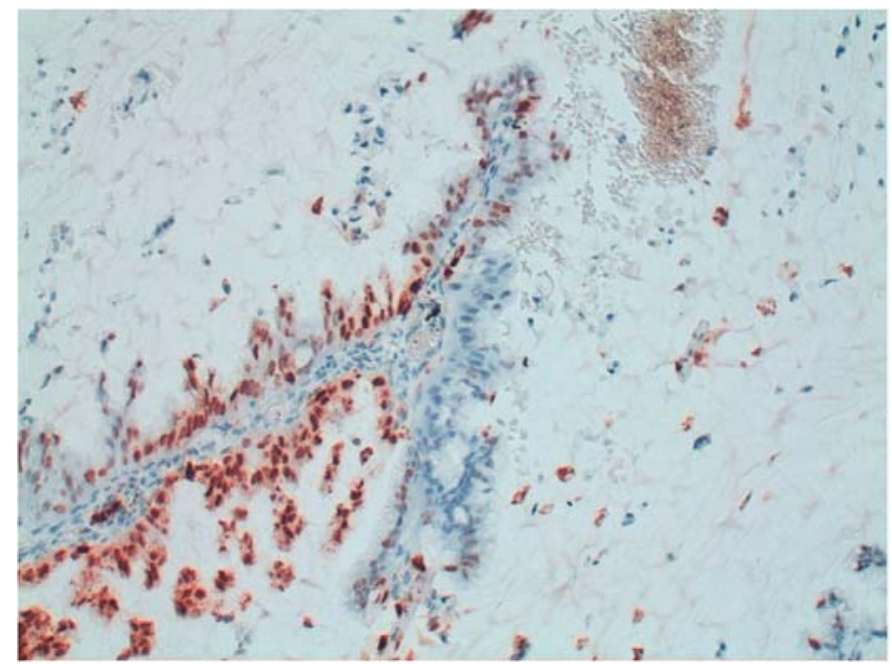

Five months later, the patient was consulted to the Emergency Department of our hospital with right secondary pneumothorax. A drainage tube was inserted to his right pleural cavity, and persistent air leakage sustained. His condition required an operation to close the pulmonary fistula. Taking the advantage of this occasion, we recommended him having additional right upper lobectomy and lymph node dissection at the same time, and he agreed with it. Operation was performed successfully, and no malignant cells were detected in the resected lung by pathological examination. 
Figure 3. Napsin A staining of the resected pulmonary nodule

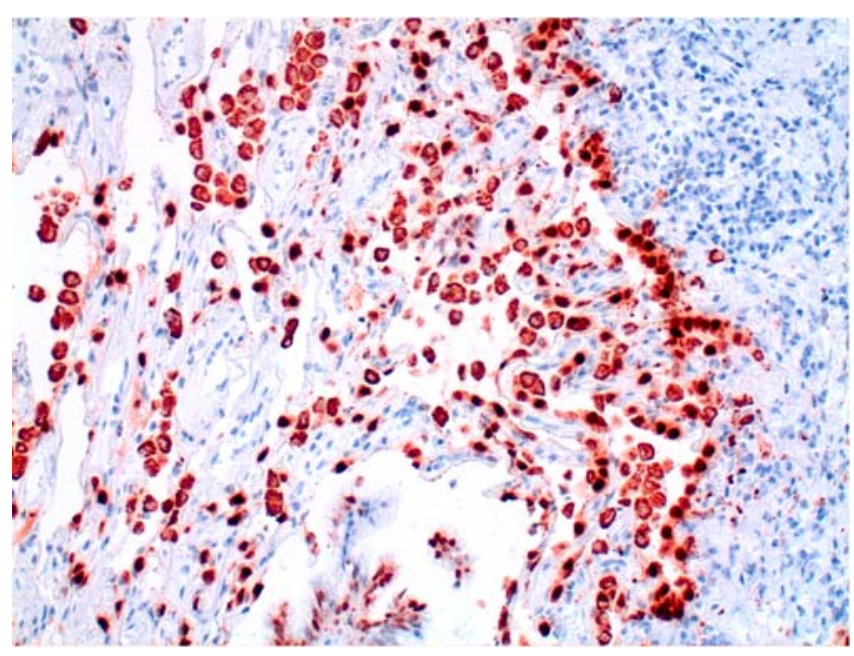

\section{Discussion}

Nowadays, Japanese enjoy one of the longest life expectancies in the world according to WHO World Health Statistics 2014, and multiple occurrences of cancers in one's lifetime are not rare. Considering that primary gastric cancer and primary lung cancer are both very common in Japanese population, it is not peculiar experience for physicians metachronously to encounter two independent cancers in the same individual.

The human lung is the organ where metastatic cancer lodges frequently from any primary sites. Further scrutinized search of the cases, however, will teach us that gastric cancer relatively less frequently choose the lung as the initial site of postoperative recurrence ${ }^{[6]}$. Many of the pulmonary sites from gastric cancer appear as lymphangiosis carcinomatosa or carcinomatous pleurisy. Solitary pulmonary metastasis of cancer was found in only $0.1 \%$ to $0.5 \%$ of patients who underwent surgery for gastric cancer ${ }^{[6,7]}$. Nakamura et al. reported that out of 13 cases who underwent pulmonary resection for the nodule after curative gastrectomy for gastric cancer (total =1,017), 8 cases were primary lung cancer and three cases were metastatic lesions from gastric cancer, one from the other organs and one benign nodule. Their (one of us is a coauthor) data also suggest the importance in preoperative diagnosis of the lesions, though the odds are greater for primary lung cancer ${ }^{[4]}$. Moreover, it has been reported that lymphatic invasion is a more important element in pulmonary metastases from gastric cancer. All of 13 cases with resected solitary pulmonary metastasis of gastric cancer in two reports, the lymphatic invasion are noticed in the primary site ${ }^{[4,8]}$. In this case, even retrospective investigation did not disclose lymphatic invasion, and it implied the risk of metastasis was small.

What is the lesson of this case? The concern on the less probability on metastatic lesion of pulmonary surgeons was not shared with the attending pathologists. Unnoticed pulmonary metastasis especially exhibited as a lymphangiosis carcinomatosa have been reported ${ }^{[9]}$, thus it is not strange for pathologists to see a metastatic lesion, especially mucinous type adenocarcinoma in the lung who have past history of "advanced" gastric cancer. The histopathological evaluation of lymphatic permeation "ly" in Japanese Gastric Classification Systems ${ }^{[3]}$ is routinely performed in Japan, but the practical value of it is not concerned clearly. It is not sure, this anecdotal experience robustly support that ly negative T2 gastric cancer has lower chance to metastasize to the lung. The checking the previous diagnosis on gastric cancer at the time of intraoperative rapid diagnosis was not thorough, just as T2 disease (categorized as advanced cancer). Actually evaluation of lymphatic permeation "ly" of gastric cancer is notoriously subjective and this evaluation is not world-wide practice. Furthermore, especially in this case, morphological feature of adenocarcinoma is non-specific at best and mucin producing pattern easily mislead the experienced pathologists to favor the metastatic. Needless to say, it may be enough to get correct diagnosis by increasing the number of staff. 
There are no reports showing that intraoperative frozen sections make false-positive diagnosis that mistakes benign for malignant, but there are $1.1 \%$ to $4.0 \%$ of false-negative diagnoses ${ }^{[1,2]}$. No researches exist which discuss the accuracy of distinguishing between gastric metastasis and primary lung cancer in frozen section and it seems that most of surgical pathologist feel difficulty in diagnosing primary organ in frozen section especially when they identified mucin producing adenocarcinoma both in the lung and in the lymph node. Several trials of intraoperative rapid immunostaining launched in some institutes, and it improved the dilemma in some cases. Even though that may be accomplished, some pulmonary marker express in gastric cancers ${ }^{[10]}$ and sharing the preoccupations of both pathologists and surgeons are essential.

Rapid immunostaining is not so popular as HE staining because it requires unconventional antibody, but its method itself have been already established according to the literature; rapid immunostaining of touch imprint cytology for sentinel lymph nodes evaluation in breast cancer for example is in practice in some institutes ${ }^{[11]}$. By combining TTF-1 and napsin A immunohistochemical analysis is a very sensitive and highly specific method for differential diagnosis of primary and metastatic lung adenocarcinoma ${ }^{[12,13]}$. About metastatic adenocarcinoma from stomach, Matoso et al. showed positive rate of TTF-1 is $1.8 \%(n=110)^{[14]}$. As mentioned above, it is very rare that gastric tumor metastasize to the lung as a solitary nodule, so there are few reports describing its immunohistochemical characteristic of napsin A. However Turner et al. showed primary gastric adenocarcinoma is not showing positive for napsin $A(n=93)^{[15]}$. In our case, the characteristic of the "primary" gastric cancer was negative for both of TTF-1 and napsin A retrospectively. Therefore positive staining of both of TTF-1 and napsin A confirms primary lung adenocarcinoma not only in our case but also in other cases.

It seems to be controversial that how immunostaining could be introduced into the routine practice of intra-operative diagnosis using frozen section because of its cost-effectiveness. In the practices of lung malignancies, however, rapid and robust intraoperative diagnosis continues to be an essential requirement, so systems and resources must be considered to accomplish this demanding vocation at both the sides of surgeons and pathologists.

\section{Funding}

This work was supported in part by the Grant-in-Aid from the Ministry of Education, Culture, Sports, Science and Technology (S-001), the Smoking Research Foundation, and National Cancer Center Cancer Research and Development Research Fund.

\section{Competing interests}

The authors declare that they have no competing interests.

\section{References}

[1] Walts AE, Marchevsky AM. Root cause analysis of problems in the frozen section diagnosis of in situ, minimally invasive, and invasive adenocarcinoma of the lung. Arch Pathol Lab Med. 2012; 136: 1515-21. PMid: 23194044. http://dx.doi.org/10.5858/arpa.2012-0042-OA

[2] Marchevsky AM, Changsri C, Gupta I, et al. Frozen section diagnoses of small pulmonary nodules: accuracy and clinical implications. Ann Thorac Surg. 2004; 78: 1755-60. PMid: 15511468. http://dx.doi.org/10.1016/j.athoracsur.2004.05.003

[3] Japanese Gastric Cancer Association. Japanese classification of gastric carcinoma: 3rd English edition. Gastric Cancer. 2011; 14: 101-12. PMid: 21573743. http://dx.doi.org/10.1007/s10120-011-0041-5

[4] Nakamura T, Homma Y, Miyata N, et al. Only surgical resection can identify the second primary lung cancer oft of the metastasis after gastric cancer surgery. Jpn J Clin Oncol. 2012; 42: 609-11. PMid: 22581909. http://dx.doi.org/10.1093/jjco/hys067

[5] Travis WD, Brambilla E, Noguchi M, et al. International Association for the Study of Lung Cancer/ American Thoracic Society/ European Respiratiory Society International Multidisciplinary Classification of Lung Adenocarcinoma. J Thorac Oncol. 2011; 6: 244-85. PMid: 21252716. http://dx.doi.org/10.1097/JTO.0b013e318206a221

[6] Kanemitsu Y, et al. Surgical resection of pulmonary metastases from gastric cancer. J Surg Oncol. 1998; 69: 147-50. http://dx.doi.org/10.1002/(SICI)1096-9098(199811)69:3<147::AID-JSO5>3.0.CO;2-H 
[7] Hanyu T, Kanda T, Matsuki A, et al. Endobronchial metastasis from adenocarcinoma of gastric cardia 7 years after potentially curable resection. World J Gastrointest Surg. 2010; 27: 270-4. PMid: 21160887. http://dx.doi.org/10.4240/wjgs.v2.i8.270

[8] Yoshida Y, Imakiire T, Yoneda S, et al. Ten cases of resected solitary pulmonary metastases arising from gastric cancer. Asian Cardiovasc Thorac Ann. 2014; 22: 578-82. PMid: 24867033. http://dx.doi.org/10.1177/0218492313513777

[9] Matsuda H, Chida K, Miwa S, et al. An autopsy case of cor pulmonale due to a pulmonary tumor embolism as the first clinical manifestation of occult gastric cancer. Nihon Kokyuki Gakkai Zasshi. 2002; 40: 910-4. PMid: 12645114.

[10] Pegolo E, Machin P, Damante G, et al. TTF-1 positivity in 2 cases of adenocarcinoma of the gastrointestinal tract. Appl Immunohistochem Mol Morphol. 2014; 22: e27-31. http://dx.doi.org/10.1097/PAI.0b013e318229e0a3

[11] Krishnamurthy S, Meric-Bernstam F, Lucci A, et al. A prospective study comparing touch imprint cytology, frozen section analysis, and rapid cytokeratin immunostain for intraoperative evaluation of axillary sentinel lymph nodes in breaset cancer. Cancer. 2009; 115: 1555-62. PMid: 19195040. http://dx.doi.org/10.1002/cncr.24182

[12] Zhang P, Han YP, Huang L, et al. Value of napsin A and thyroid transcription factor-1 in the identification of primary lung adenocarcinoma. Oncol Lett. 2010; 1: 899-903. PMid: 22966403.

[13] Ye J, Findeis-Hosey JJ, Yang Q, et al. Combination of napsin A and TTF-1 immunohistochemistry helps in differentiating primary lung adenocarcinoma from metastatic carcinoma in the lung. Appl Immunohistochem Mol Morphol. 2011; 19: 313-317. PMid: 21464700. http://dx.doi.org/10.1097/PAI.0b013e318205b059

[14] Matoso A, Singh K, Jacob R, et al. Comparison of thyroid transcription factor-1 by two monoclonal antibodies in pulmonary and non-pulmonary primary tumors. Appl Immunohistochem Mol Morphol. 2010; 18: 142-149. PMid: 19887917. http://dx.doi.org/10.1097/PAI.0b013e3181bdf4e7

[15] Turner BM, Cagle PT, Sainz IM, et al. Napsin A, a new marker for lung adenocarcinoma, is complementary and more sensitive and specific than thyroid transcription factor 1 in the differential diagnosis of primary pulmonary carcinoma; evaluation of 1674 cases by tissue microarray. Arch Pathol Lab Med. 2012; 136: 163-171. PMid: 22288963. http://dx.doi.org/10.5858/arpa.2011-0320-OA 